\title{
Diagnosing the initial stages from solid to plasma phase for dense plasma explosions
}

\author{
I. Fitilis ${ }^{1,2}$, A. Skoulakis ${ }^{1,2}$, E. Kaselouris ${ }^{1,2,3,{ }^{*}}$, I.K. Nikolos ${ }^{3}$, E. Bakarezos ${ }^{1,2}$, N.A. \\ Papadogiannis $^{1,2}$, V. Dimitriou ${ }^{1,2}$ and M. Tatarakis ${ }^{1,2}$ \\ ${ }^{1}$ Centre for Plasma Physics \& Lasers - CPPL, Technological Educational Institute of Crete-TEI of Crete \\ Chania \& Rethymnon, Greece \\ ${ }^{2}$ School of Applied Sciences, Technological Educational Institute of Crete- TEI of Crete \\ Chania, Greece \\ ${ }^{3}$ School of Production Engineering \& Management, Technical University of Crete, \\ Chania, Greece \\ E-mail: fitilis@chania.teicrete.gr, skoulakis@chania.teicrete.gr, \\ v.kaselouris@chania.teicrete.gr, jnikoloddpem.tuc.gr, \\ bakarezosestaff.teicrete.gr, npapadogiannisestaff.teicrete.gr, \\ dimvasi@chania.teicrete.gr, m.tatarakis@chania.teicrete.gr \\ (corresponding author)
}

\begin{abstract}
This study is focused on the understanding of the initial stages of explosion of dense plasmas as the phase changes from solid to plasma. Experiments are carried out using a Z-pinch pulsed powered device implemented in a mode of producing a peak current of $35 \mathrm{kA}$ with a rise time (10\%-90\%) of $60 \mathrm{~ns}$. Experimental results for the expansion dynamics of the exploded plasma are obtained from laser probing diagnostics such as a modified Fraunhofer diffraction probe method and also shadowgraphic and interferometric techniques. Additionally, time-integrated optical imaging is used for the visualization of the matter dynamics. A coupled transient electromagnetic-thermal-structural multiphysics computational model based on the Finite Element Method is developed with temperature-dependent material properties in order to provide valuable insights for important quantitative parameters such as temperature, density and expansion rate of the exploded material. In order to simulate the matter's response, a strength material model (Johnson-Cook) along with an equation of state (Gruneisen) are used. Comparison of experimental vs numerical results of the expansion rates of the mater and the phase change characteristics of the exploded matter gives satisfactory agreement.
\end{abstract}

First EPs Conference on Plasma Diagnostics - $1^{\text {st }}$ ECPD

14-17 April 2015,

Villa Mondragone, Frascati (Rome) Italy

\section{Presenter}




\section{Introduction}

The physical processes that take place at the initial stages of electrical exploding wire play an important role in plasma formation in pulsed-power Z-Pinch experiments $[1,2]$. The early time dynamics of the processes involved in the explosion of the wire has been proven to be important for the development of Magneto-Hydrodynamic (MHD) instabilities of the Z-pinch plasma $[3,4]$. In experiments of wire arrays with current prepulse the single wire dynamics presents a significant interest for dealing with the problem of stabilization of the implosion [5].

In order to investigate the crucial stages of the phase changes from thermoelastic to melting and plasma regimes thick metallic copper wires have been used in this study. For such wires, electrical charges flow through the skin depth which plays an important role for the exploding dynamics [6]. Laser probing diagnostics such as schlieren, interferometric and diffraction imaging techniques have been used for the measurement of the wire dynamics at the initial stages of the explosion. Moreover, a coupled transient electromagnetic-thermal-structural computational model, based on the Finite Element Method (FEM), is developed in order to provide valuable insights for important quantitative parameters such as temperature, density and expansion rate of the exploded material. The combination of laser probing diagnostics and multiphysics FEM simulations constitutes a powerful tool for analyzing and understanding the initial stages of plasma formation in the wire.

\section{Experimental set-up}

Experiments are carried out using a Z-pinch pulsed powered device implemented in a mode of producing a peak current of $35 \mathrm{kA}$ with a rise time (10\%-90\%) of $60 \mathrm{~ns}$. The Z-pinch pulsed power device consists of a Marx bank of $600 \mathrm{~J}$ energy capacity, a water-filled pulse forming line (PFL) and a self-breaking SF6 switch. The copper wire of $300 \mu \mathrm{m}$ diameter and $15.2 \mathrm{~mm}$ length is placed in a vacuum chamber evacuated at $10^{-4} \mathrm{mbar}$. The wire is fixed by soldering it to the conical shaped copper electrodes. A V-dot probe and a Rogowski groove are used to measure the derivatives of the voltage at the PFL and of the current passing through the wire respectively. The second harmonic of a SBS-compressed Nd:YAG, Q-switch laser (EKSPLA, SL312), with 150 ps pulse duration, is used for the schlieren, interferometric and diffraction imaging laser probing techniques. This gives the ability of ns time resolved tracing of the explosion stages. The imaging of the Fraunhofer diffraction at the focus of a lens is employed as a method to determine the expansion of the wire at times before plasma formation [7]. For the schlieren imaging a knife-edge oriented parallel to the wire is used at the focal length of the imaging lens and the formation of coronal plasma is revealed from the bright light appeared at the same side of the wire as that the knife is placed [8]. A Mach-Zehnder interferometer in finite-fringe mode is also developed and used for plasma density measurements. 


\section{Finite Element simulation}

A 3D multiphysics simulation based on FEM is developed. Maxwell equations (eddycurrent approximation) are solved using finite elements for the wire coupled with a Boundary Element Method (BEM) for the surrounding vacuum. Moreover, the skin depth effect for the wire is also taken into account. When the electromagnetic fields have been computed, the Lorentz force $\mathbf{F}=\mathbf{j} \times \mathbf{B}$, where $\mathbf{j}$ is the current density and $\mathbf{B}$ is the magnetic field, is evaluated at the nodes and added to the mechanical solver, which computes the deformation of the wire. Furthermore, the Joule heating power term $\left(\mathrm{j}^{2} / \sigma\right.$, where $\sigma$ the electrical conductivity) is added to the thermal solver, so as to update the temperature [9].

In order to properly simulate the magneto-hydrodynamic response of metal: the hydrodynamic behavior is taken into account by using analytical Gruneisen equation of state [10], the deviatoric behavior is taken into account using Johnson-Cook [11] strength material model and the electrical conductivity versus temperature and density is computed using Burgess equation of state [12]. For the mechanical response, the Johnson-Cook model is coupled with the Gruneisen equation of state. The Johnson-Cook material model takes into account the effect of plastic strain, strain rate and temperature rise. Temperature dependent properties of thermal expansion, thermal conductivity and specific heat, as well as the latent heat of melting are also taken into account. Regarding the boundary conditions, the ends of the wire are fixed at environmental temperature $\left(27^{\circ} \mathrm{C}\right)$. An important aspect of the developed simulation is that the Lagrangian mesh is appropriately refined in order to accurately simulate the dynamic phase changes of matter in the region of the skin depth. The loading source term is the alternating current as measured and recorded during the real experiments.

\section{Results and discussion}

The explosion of the initially solid metal wire and its intermediate phases are investigated, up to the plasma formation, in this study. In order to investigate the wire diameter dynamics before plasma formation, a modified Fraunhofer diffraction method is implemented with high accuracy in measuring the wire width. The fringes pattern at the focus of a lens is recorded before the explosion and at a time defined form the delay unit. The focal spot of the laser probe beam was shifted just out of the CCD's frame of the camera in order to reveal the second and higher order fringes at the image. The line out of the image along the diffraction axis is used for the measurement of the wire's diameter and consequently the evaluation of the expansion at the time of the snapshot. Fig.1 illustrates the diffraction pattern of the measurement of the wire's diameter at $140 \mathrm{~ns}$ from the current start, just before plasma formation starts. At this time plasma has not yet been formed as confirmed by the simultaneously obtained interferogram. The measured diameter compared with the initial measurement shows an expansion of $7.4( \pm 0.8)$ $\mu \mathrm{m}$. At that time the simulation gives an expansion of $7( \pm 0.2) \mu \mathrm{m}$. An average radial expansion rate of $65 \mathrm{~m} / \mathrm{s}$ results from experimental measurements, while a radial expansion rate of $60 \mathrm{~m} / \mathrm{s}$ is computed in the simulations. 


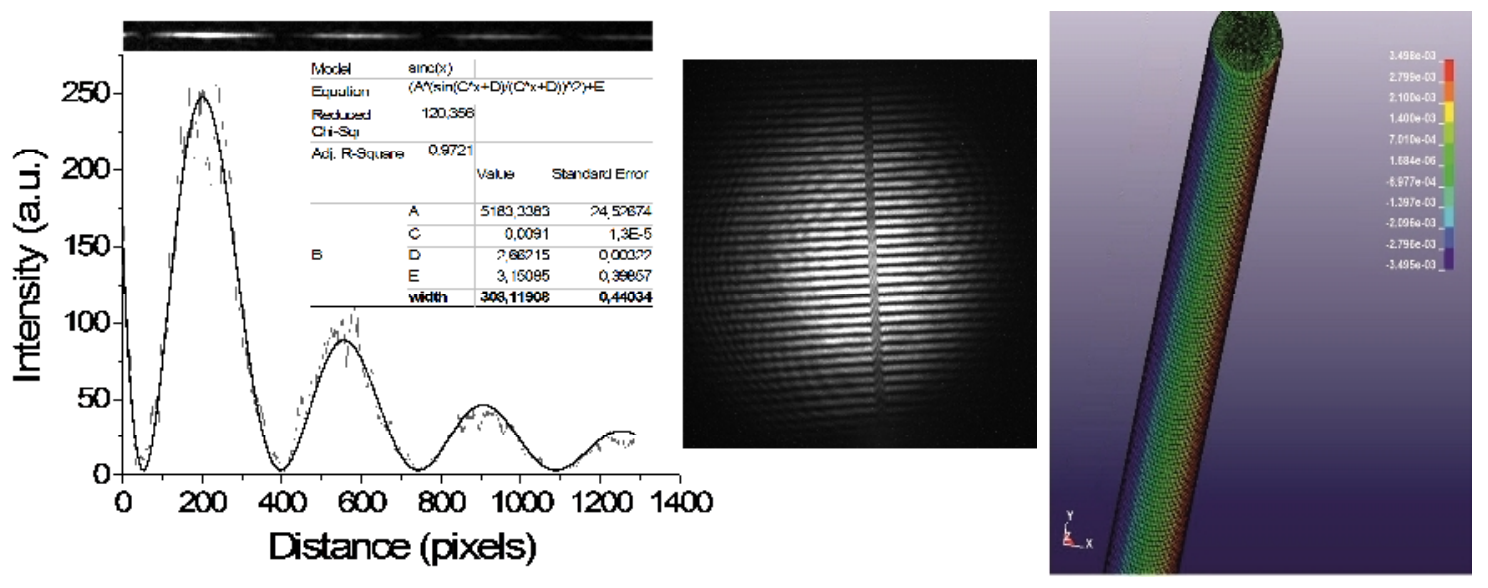

Figure 1: Left: Laser probe diffraction pattern (top) and the lineout intensity plot along the axis of the fringes (bottom) at $140 \mathrm{~ns}$ from the current start. Middle: Interferogram at the same time showing no plasma formation. Right: Displacement of the $\mathrm{x}$-axis $(\mathrm{mm})$ of the wire at the same time(FEM simulation).

In order to experimentally investigate the plasma formation at early times and especially to elucidate information about the initial time where the plasma formation starts, a series of schlieren and interferometric images were taken at different times from the current start. Fig.2 presents an interferogram as well as a schlieren image taken simultaneously at $150 \mathrm{~ns}$ from the current start showing the initiation of the coronal plasma formation at the bottom of the wire (cathode). Fig.3 presents simulation results at the same time showing the computed temperature and density distributions from a cross-section of the wire. A temperature of $3580{ }^{\circ} \mathrm{C}$ and a mass density of $3.5 \mathrm{~g} / \mathrm{cm}^{3}$ at the outer part are predicted by the code. Although the plasma initial conditions are now under investigation, these values can be considered to be in the expected window range [13]. Fig.4 shows an interferogram and a schlieren image at $220 \mathrm{~ns}$ from the current start which clearly shows that plasma has been formed along the wire at this time. From the interferogram a maximum coronal plasma electron density of $3 \times 10^{18} \mathrm{~cm}^{-3}$ at a radius of 285 $\mathrm{m}$ can be calculated [3]. The coronal plasma surrounds the dense plasma core where the probe laser beam cannot propagate due to refraction in the steep plasma transverse density gradients [3]. It should be pointed out here that especially at early times, the formation of the coronal plasma can coexist with a neutral atomic gas surrounding the wire. On the images of Fig. 4 the decrease of the refractive index due to coronal plasma is dominated near the wire as can be seen from the resulting upwards bending of the interferogram fringes. This is also confirmed by the schlieren image which shows a bright light mainly at the left side of the wire, the side which the "schlieren stop" cuts-off the probe laser focal spot. Contrarily, at the other side of the wire there is dark, except for some places at the bottom and the middle of the wire. These suggest that the gas is significantly ionized to plasma which is subject of further investigation. The depicted numerical simulation results in Fig.5 predict the temperature and the density of the outer part of the wire to be $5200{ }^{\circ} \mathrm{C}$ and $1.7 \mathrm{~g} / \mathrm{cm}^{3}$ respectively (while copper's solid density is $8.96 \mathrm{~g} / \mathrm{cm}^{3}$ ). These values are in the range of experimental measured values for strongly coupled dense copper plasma found in the literature $[14,15]$. Thus, the simulation indicates that coronal plasma has been formed at this time in agreement with the experiment. 


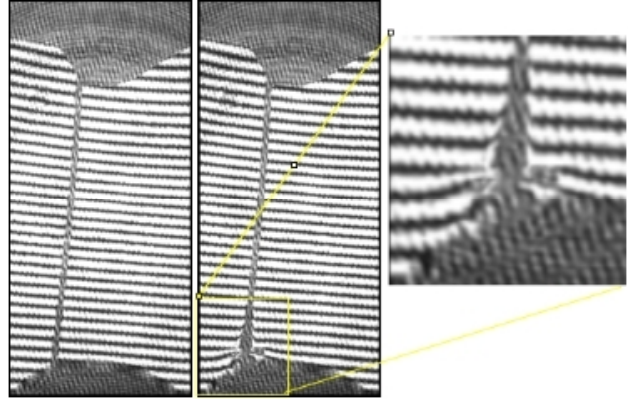

a

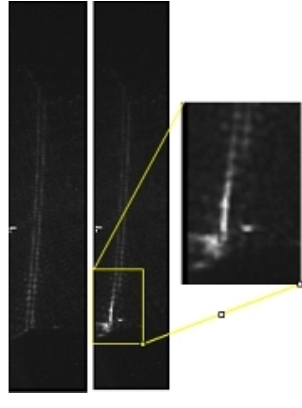

b

Figure 2: Interferograms (a) and schlieren images (b) before the shot (left) and at $150 \mathrm{~ns}$ after current start (right) showing the initiation of corona plasma formation at the bottom of the wire.
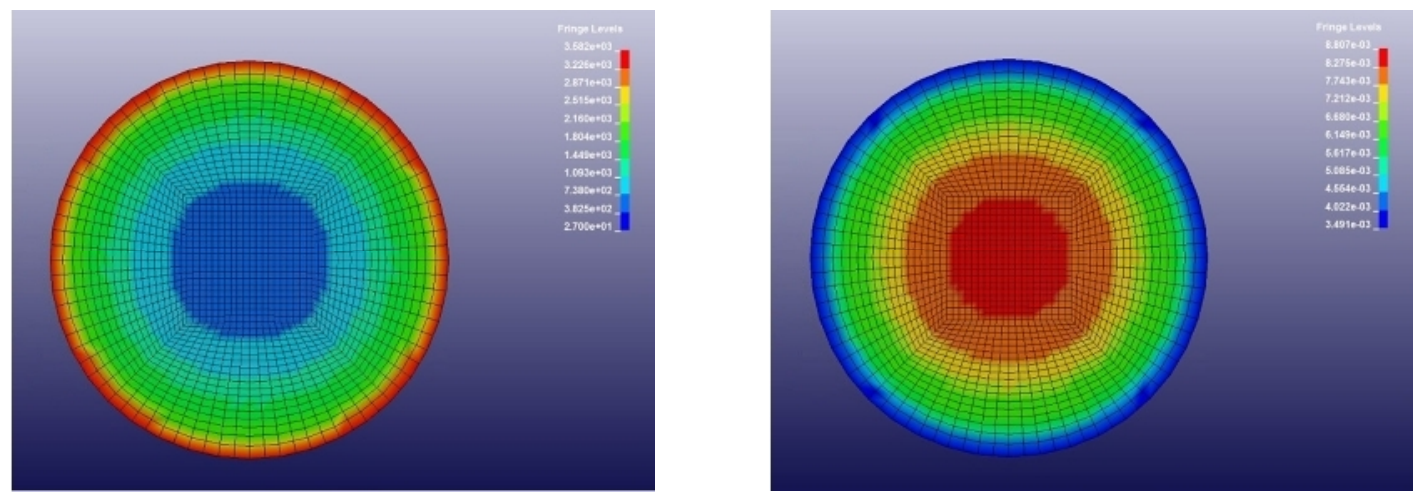

Figure 3: Finite element numerical results for temperature (left, ${ }^{\circ} \mathrm{C}$ ) and density (right, $\mathrm{kg} / \mathrm{cm}^{3}$ ) from a cross section of the wire at $150 \mathrm{~ns}$ from current start.

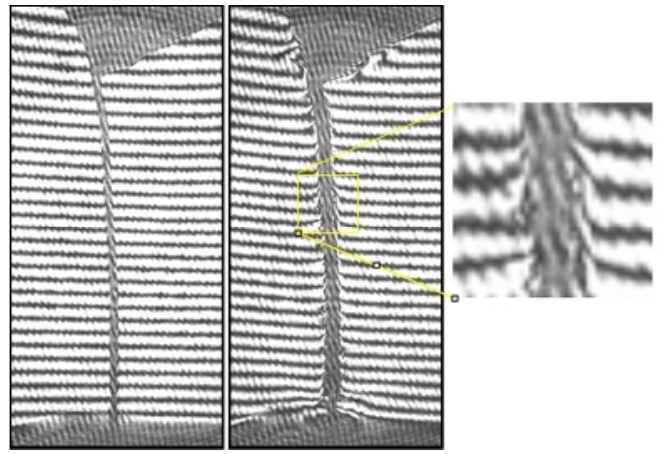

a

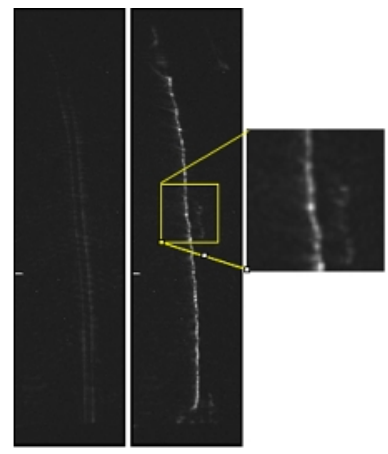

b

Figure 4: Interferograms (a) and schlieren images (b) before the shot (left) and at $220 \mathrm{~ns}$ after current start (right).
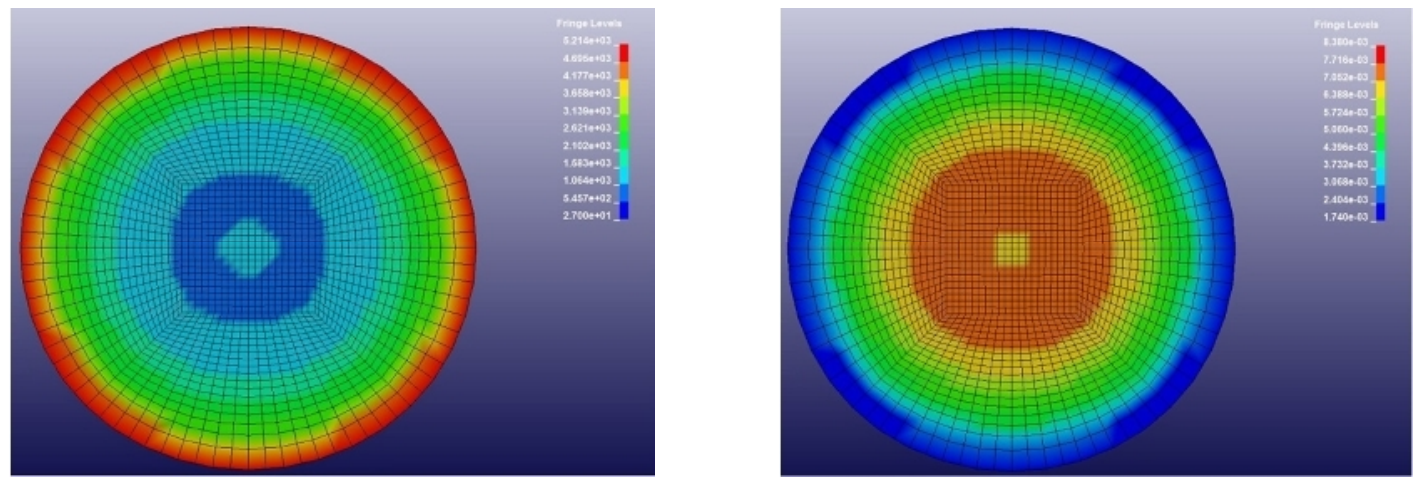

Figure 5: Finite element numerical results for temperature (left, ${ }^{\circ} \mathrm{C}$ ) and density (right, $\mathrm{kg} / \mathrm{cm}^{3}$ ) from a cross section of the wire at $220 \mathrm{~ns}$ from current start. 
The preliminary investigation presented here, shows satisfactory agreement of experimental vs FEM results concerning both the initial times of wire's expansion (before plasma formation) as well as the coronal plasma formation. The combination of the multiphysics FEM model and the experimental method is capable to describe the wire expansion dynamics. Further coupled-field numerical simulations that use a multiphase equation of state instead of the analytical Gruneisen along with new experiments are under development.

\section{Acknowledgments}

The authors acknowledge financial support through the Action "National Research Infrastructure for HiPER" MIS 376841 (co-funded by the European Union and Hellenic National funds within the Operational Programme "Competitiveness and Entrepreneurship").

\section{References}

[1] M.G. Haines, A review of the dense Z-pinch, Plasma Phys. Control. Fusion 53, 093001 (2011).

[2] D.A. Hammer and D.B. Sinars, Single-wire explosion experiments relevant to the initial stages of wire array z pinches, Laser Part. Beams 19, 377-391 (2001).

[3] M. Tatarakis et al., Optical probing of fiber z -pinch plasmas, Phys. Plasmas5, 682 (1998).

[4] V.I. Oreshkin et al., Wire explosion in vacuum: Simulation of a striation appearance, Phys. Plasmas 11, 4771-76 (2004).

[5] H. Calamy et al., Use of microsecond current prepulse for dramatic improvements of wire array Zpinch implosion, Phys. Plasmas 15, 012701 (2008).

[6] D.P. Wall et al., Influence of the skin effect and current risetime on the fragmentation of wires by pulsed currents, J. of Appl. Phys. 98, 023304 (2005).

[7] S.A. Khodier, Measurement of wire diameter by optical diffraction, Opt. \& Laser Technol.36, 63-67 (2004)

[8] S.I. Tkachenko et al., Distribution of matter in current-carrying plasma and dense core of the discharge channel formed upon electrical wire explosion, Plasma Phys. Rep. 35, 734-753 (2009).

[9] G. Le Blanc et al., Ramp wave compression in a copper strip line: comparison between MHD numerical simulations (LS-DYNA) and experimental results (GEPI device), 10th International LSDYNA Conference, 2008.

[10] K. Nagayama, Introduction to the Grüneisen Equation of State and Shock Thermodynamics, Kindle Edition.

[11] G.R Johnson and W.H. Cook, Fracture characteristics of three metals subjected to various strains, strain rates, temperatures and pressures, Eng. Fract. Mech 21, 31-48 (1985).

[12] T. Burgess, Electrical resistivity model of metals, $4^{\text {th }}$ International Conference on Megagauss Magnetic-field generation and related topics, Santa Fe, NM, 1986.

[13] B.I. Cho et al., Electronic Structure of Warm Dense Copper Studied by Ultrafast X-Ray Absorption Spectroscopy, Phys. Rev. Lett. 106, 167601 (2011).

[14] A.W. Desilva and H.J. Kunze, Experimental study of the electrical conductivity of strongly coupled copper plasmas, Phys. Rev. E 49, 4448 (1994).

[15] M.P. Desjarlais, Practical improvements to the Lee-More Conductivity near the metal-insulator transition, Contrib. Plasma Phys. 41, 267 (2001). 